\title{
Dynamics of a microcapillary discharge plasma using a soft x-ray laser backlighter
}

\author{
M. C. Marconi, ${ }^{1, *}$ C. H. Moreno, ${ }^{1, *}$ J. J. Rocca, ${ }^{1}$ V. N. Shlyaptsev, ${ }^{2}$ and A. L. Osterheld ${ }^{3}$ \\ ${ }^{1}$ Department of Electrical and Computer Engineering, Colorado State University, Fort Collins, Colorado 80523 \\ ${ }^{2}$ Department of Applied Sciences, University of California Davis-Livermore, Livermore, California 94551 \\ ${ }^{3}$ Livermore National Laboratory, Livermore, California 94551
}

(Received 22 February 2000)

\begin{abstract}
We have used the new technique of soft $\mathrm{x}$-ray laser shadowgraphy in combination with traditional plasma emission spectroscopy and theoretical modeling to study the dynamics of a plasma column created by a discharge through a $380 \mu \mathrm{m}$ diameter evacuated microcapillary. The transient microcapillary plasma was imaged with high-spatial and temporal resolution using a tabletop discharge pumped 46.9-nm laser backlighter. Model computations show that the sharp boundary observed between the absorbent and transparent regions of the shadowgrams is defined by the spatial distribution of weakly ionized ions that are strongly photoionized by the probe laser. The plasma was observed to rapidly evolve from an initially nonuniform distribution into a column with good azimuthal symmetry and minimum density on axis [computed electron density on axis $n_{e}$ $\left.=(1-3) \times 10^{19} \mathrm{~cm}^{-3}\right]$. This concave electron density profile constitutes a plasma waveguide for laser radiation. Heated solely by Joule dissipation from relatively small excitation currents $(1.5 \mathrm{kA})$, this dense plasma reaches substantial electron temperatures of $T_{e}=15-20 \mathrm{eV}$ as a result of the absence of significant hydrodynamic losses and reduced radiation losses caused by large spectral line opacities. The results illustrate the potential of tabletop soft $\mathrm{x}$-ray lasers as a new plasma diagnostic tool.
\end{abstract}

PACS number(s): 52.80.- s, 52.65.-y, 52.40.Fd, 42.55.Vc

\section{INTRODUCTION}

Discharges in gas-filled and evacuated capillary channels have a long history of use in applications [1-21], that includes the successful development of high-gain tabletop soft X-ray lasers [10-14]. Recently, plasmas with concave electron-density profiles created in microcapillary channels by either discharge [15-21] or laser excitation [19,22,23] have received attention as plasma waveguides for intense laser radiation. Plasma waveguides are of significant interest for the development of soft X-ray lasers and particle accelerators [15-28]. Optical-field ionization and transient heating of capillary plasmas with intense ultrashort pulse lasers have resulted in the observation of soft x-ray amplification following plasma recombination $[22,23]$ and collisional excitation, respectively [18].

The recent advent of saturated table-top soft x-ray lasers $[11,14,29-31]$ has opened the possibility of utilizing soft $\mathrm{x}$-ray laser beams to probe a wide variety of high-density plasmas of large dimension. Their short wavelength, high brightness, short pulse duration, and high degree of collimation makes soft $\mathrm{x}$-ray lasers ideal sources to perform shadowgraphy studies in dense plasmas [32-34]. We have recently reported the use of a tabletop soft $\mathrm{x}$-ray laser to obtain shadowgrams of a capillary discharge plasma [34]. In this paper we discuss the experiment in detail, and analyze the results in combination with traditional plasma emission spectroscopy and extensive theoretical modeling to gain understanding of the dynamics of a plasma created by a discharge through a $380 \mu \mathrm{m}$ diameter evacuated polyacethal microcapillary. Many of the recent experiments and theoretical studies in fast capillary discharges have been concerned with plas-

\footnotetext{
*Permanent address: Departamento de Fisica, FCEyN, University of Buenos Aires, Buenos Aires, Argentina.
}

mas that rapidly contract under the influence of the $J \times B$ ponderomotive or Lorentz force $[6,9,11]$. In spite of some important specific distinctions caused by the close proximity of the walls, it is valid to classify these discharges as a kind of $Z$ pinch. In contrast, in the microcapillary plasma that is the subject of this paper the Lorentz force has negligible effect due to a lower peak current $(\approx 1.5 \mathrm{kA}$ instead of tens of $\mathrm{kA}$ ). This, and the strong coupling between the plasma and the polyacethal wall, cause a different plasma heating, a contraction behavior that distinguishes this type of microcapillary discharges from $Z$ pinches and classifies it into a wallsustained plasma. Such discharges in evacuated capillaries can create a concave electron-density profile that has been observed to provide optical guiding for tightly focused laser beams over distances much longer than the Rayleigh length [15-17].

The next section discusses the setup used in the shadowgraphy measurements, and Sec. III presents the experimental results. Section IV includes the model calculations and a discussion of the dynamics of the microcapillary plasma based on the experimental and theoretical results.

\section{EXPERIMENTAL SETUP}

The microcapillary plasma subject of this paper was generated by exciting a $380-\mu \mathrm{m}$-diameter, 8 -mm-long evacuated channel in polyacetal $\left(\mathrm{CH}_{2} \mathrm{O}\right)_{n}$ with a current pulse of 1.5 kA-peak amplitude and 155-ns-half-period duration. Measurements were also conducted for plasmas generated in capillary channels $500 \mu \mathrm{m}$ diameter. The plasma was generated from material ablated from the capillary walls by the current pulse. Prior to excitation the pressure in the vacuum chamber containing the capillary discharge was lower than 1 $\times 10^{-5}$ Torr. To record the shadowgrams the capillary discharge plasma channel was illuminated on axis by a lowdivergence beam of 46.9-nm-wavelength radiation produced 


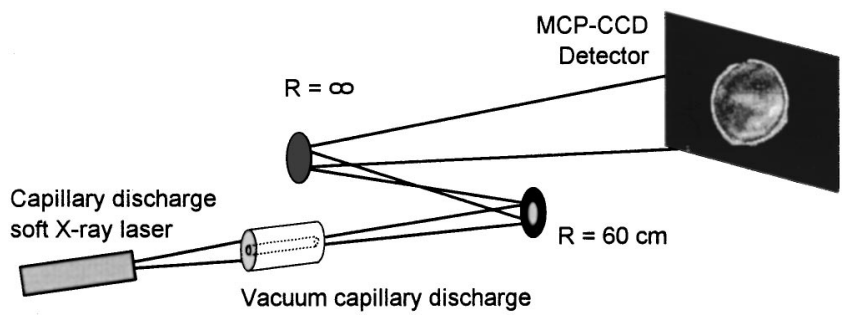

FIG. 1. Schematic representation of the setup used to obtain shadowgrams of a microcapillary plasma using a 46.9-nm-tabletop laser as backlighter.

by a tabletop Ne-like Ar capillary discharge laser. The generation of 46.9-nm-laser pulses from tabletop capillary discharge soft $\mathrm{x}$-ray amplifiers has been discussed in previous publications [10-14]. The laser used in this experiment emits pulses of 0.6 to 0.7 ns full width at half-maximum duration and energy up to $25 \mu \mathrm{J}$ in a single strong laser line [13]. The laser pulses were produced exciting with a fast current pulse a 16.4-cm-long, 4-mm-diameter polyacetal capillary channel filled with Ar gas at a pressure of 600 mTorr. The current pulses had a peak amplitude of approximately $37 \mathrm{kA}$ and a first half-period of about $70 \mathrm{~ns}$. Lasing in the $3 p-3 s J=0$ -1 line of Ne-like Ar occurs approximately $39 \mathrm{~ns}$ after the onset of the current, following the generation of an elongated Ar plasma column by a rapid compression. The laser beam divergence is approximately $5 \mathrm{mrad}$.

The imaging setup used to record the shadowgrams is schematically illustrated in Fig. 1. The microcapillary discharge was placed at $60 \mathrm{~cm}$ from the exit aperture of the soft $\mathrm{X}$-ray laser. This distance was selected to comfortably overfill the capillary channel aperture with the soft $\mathrm{x}$-ray laser beam, with the purpose of ensuring a relatively uniform illumination of the plasma. The capillary discharge plasma was imaged into a two-dimensional soft $\mathrm{x}$-ray sensitive detector using a $60 \mathrm{~cm}$ radius of curvature concave iridium-coated mirror. This concave mirror was positioned at $31.3 \mathrm{~cm}$ from the exit of the capillary. A flat iridium-coated mirror was used between the curved mirror and the detector to relay the image. The reflectivity of the iridium mirrors used in this experiment was estimated to be about $10 \%$ at $46.9 \mathrm{~nm}$ from the $\sim 100 \times$ loss of laser intensity measured by inserting the mirrors in the laser path. The detector consisted of a microchannel-plate followed by a phosphor screen, an image intensifier, and a charged-coupled device array (CCD). The CCD consists of a $1024 \times 1024$ array of $24.8 \mu \mathrm{m}$ pixels. A thin Al film was evaporated on the phosphor screen to avoid the detection of visible plasma radiation. The microchannel plate was gated during about $5 \mathrm{~ns}$ to differentiate the laser radiation from the long lasting $(>100 \mathrm{~ns})$ short wavelength radiation emitted by the microcapillary discharge. The curved mirror imaged the capillary plasma on the detector plane with a magnification of $\sim 24 \times$. The spatial resolution of the entire imaging system was determined to be approximately $5 \mu \mathrm{m}$ in the object plane by imaging an array of 25 $\mu \mathrm{m}$ diameter holes placed at the exit plane of the capillary channel. This measurement also allowed for a direct experimental calibration of the magnification of the imaging system.

\section{EXPERIMENTAL RESULTS}

Figure 2 shows a sequence of soft x-ray shadowgrams that describe the evolution of the plasma created in a 380$\mu \mathrm{m}$-diameter capillary channel by the discharge current pulse. The time delay between the initiation of the current pulse and each of the shadowgrams is indicated. The first image in the sequence was obtained by propagating the soft $\mathrm{X}$-ray laser pulse through the capillary before the initiation of the discharge current pulse, and therefore corresponds to the situation in which no plasma is present. A relatively uniform illumination of the capillary channel is observed. The second (26 ns) and third images (39 ns) are illustrative of the initial phase of the plasma evolution, corresponding to times shortly after the breakdown. Dense regions of ablated material that strongly absorb the soft x-ray laser radiation are observed to develop at a few locations on the wall perimeter. This indicates that during the initial phase of the discharge a significant fraction of the current flow is predominately localized in a few surface discharge channels. However, as the current increases and the plasma expands towards the evacuated center of the capillary, these initial nonuniformities decrease, and the boundary between the absorbing and transparent regions of the plasma is observed to acquire a remarkable symmetry. The shawdograms taken at times after approximately $80 \mathrm{~ns}$ from the initiation of the current pulse, show a very symmetric annular absorbing region that rapidly expands towards the axis of the discharge. The rate of expansion of the absorbing region towards the capillary axis can be inferred from the measured variation of its inner diameter as a function of time. The velocity of the boundary is $5 \times 10^{4}$ and $1 \times 10^{5} \mathrm{~cm} / \mathrm{s}$ at 50 and $100 \mathrm{~ns}$ after the onset of the current pulse, respectively. As discussed below, this velocity is not the rate of expansion of the plasma, but rather that of a region containing a large concentration of weakly ionized species that attenuate the laser beam by photoionization. At about $140 \mathrm{~ns}$ after the onset of the discharge, near the end of the first half-cycle, the central region of the plasma starts to significantly absorb the soft x-ray laser beam. Shadowgrams obtained for a 500- $\mu \mathrm{m}$-diameter capillary showed a qualitatively similar evolution of the plasma, described by a rapid transition from an initially asymmetric plasma into a highly symmetric plasma column with minimum density on axis.

The same imaging setup used in the shadowgraphy experiment was also employed to record the evolution of the spatial distribution of the vacuum ultraviolet (VUV) and soft $\mathrm{x}$-ray radiation self-emitted by the microcapillary plasma. Figure 3 shows a sequence of time resolved cross sections of the intensity distribution of the self-emitted plasma radiation obtained gating the MCP detector. Shortly after the onset of the discharge the emitted radiation is observed to be weak and mainly concentrated near the capillary walls, where the plasma is created by ablation and ionization of wall material by the discharge. Plasma emission also appears to emanate at this time from the central part of the capillary. Nevertheless, the emission observed as originating from the axial region at the earliest times in Fig. 3, can be, in part, an artifact caused by radiation originating from outer regions of the plasma at different axial locations in the capillary that, unlike the exit aperture of the capillary, are out of focus at the image plane. A simple ray-optics calculation can show that imaging of a cylindrically symmetric radiant volume of annular cross section with our optical system results in intensity patterns that 

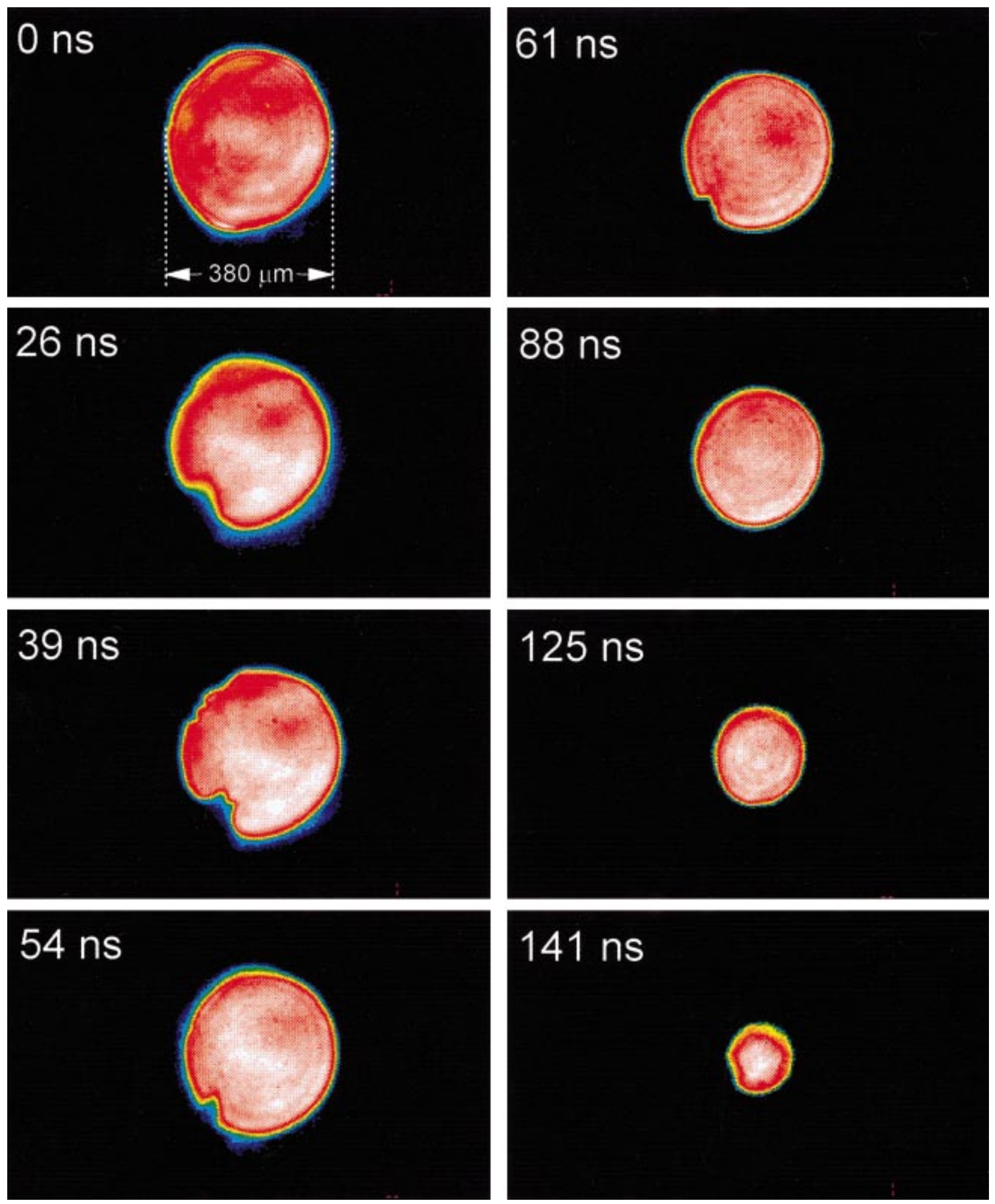

FIG. 2. (Color) Sequence of on-axis shadowgrams describing the evolution of the microcapillary plasma. The time of each shadowgram, with respect to the initiation of the current pulse, is indicated. The diameter of the microcapillary is $380 \mu \mathrm{m}$ and the length $8 \mathrm{~mm}$. Lighter regions represent less absorption of the soft x-ray laser beam.

correspond well with those of the earlier times in Fig. 3. Nevertheless, as the discharge evolves the plasma emission is observed to shift to the central region of the capillary and greatly increase in intensity.

The spectral distribution of the plasma self-emission in the 50-120 nm region was recorded using a 1-m-focal length normal-incidence vacuum spectrograph with a 600 line/mm diffraction grating and a MCP intensified diode array detector. For these measurements the capillary discharge was placed directly in front of the spectrograph. Spectra with a time resolution of about $10 \mathrm{~ns}$ were obtained by gating the intensifier. The sequence of VUV spectra displayed in Fig. 4 shows emission from carbon ions with a degree of ionization ranging from $\mathrm{C}$ II to $\mathrm{C}$ IV and from oxygen ions ionized up to 

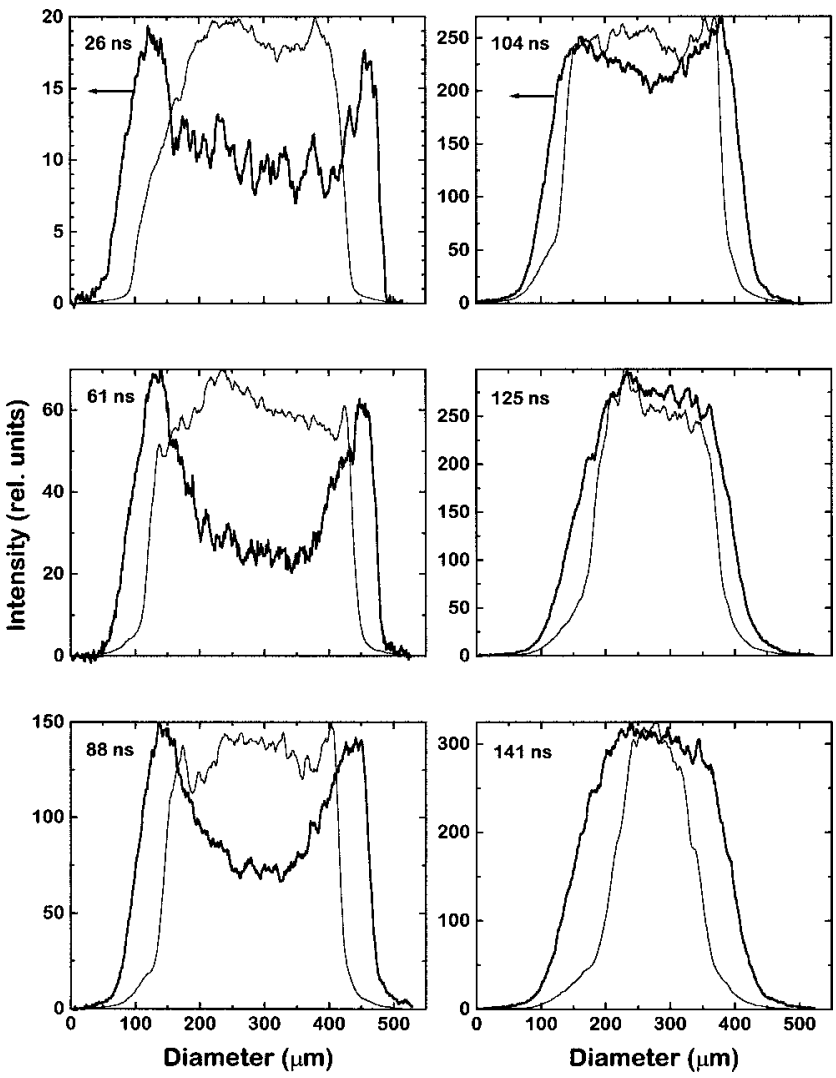

FIG. 3. Thick line: sequence of cross sections of time-resolved on-axis images of the VUV/soft x-ray self-emission of the microcapillary plasma. Thin line: corresponding profiles of the transmitted soft x-ray laser intensity obtained from the shadowgrams (the intensity was normalized to facilitate comparison of the profiles). The time of each profile, with respect to the initiation of the current pulse, is indicated. The earlier emission profiles, that were obtained after a sequence of about 100 shadowgram shots, are wider than the initial 380- $\mu \mathrm{m}$-capillary-bore diameter due to an increase in the bore diameter resulting from ablation by the discharge pulses.

O VI. The observation of emission from $\mathrm{C}$ II ions, that have an ionization energy of $24.4 \mathrm{eV}$, and from excited states of $\mathrm{C}$ III and C IV that have ionization energies that are lower than the $26.5 \mathrm{eV}$ energy of the laser photons, suggests that in the colder regions of the plasma photoinization of weakly ionized atoms is responsible for an important part of the observed laser beam attenuation. The presence of $\mathrm{O}$ VI lines indicates that the electron temperature in the hottest region of the plasma reaches at least $15 \mathrm{eV}$.

\section{MODEL RESULTS AND DISCUSSION}

The evolution of the microcapillary plasma was modeled with the one-dimensional hydrodynamic-atomic code RADEX [35,36]. The physical model of the evacuated microcapillary discharge of this paper is similar to that previously used to analyze gas-filled capillary soft $\mathrm{x}$-ray lasers and laser-produced plasmas [9-11,35-37], and while it does not include the detailed computation of population inversions, it has a similar degree of complexity. The model includes hydrodynamics equations, Maxwell's equations, and the atomic kinetics of the different atomic species present in the plasma. For the modeling of the evacuated microcapillary plasma the numerical mesh has to be inhomogeneous and able to repro- duce a very broad density spread covering six to eight orders of magnitude from the initially solid material to the relatively low-density vapors that, after compression, become a dense plasma. Besides the small grid cell sizes and the strict Courant time step criteria requirements associated with them [38], another restriction results from the short-time scales mandated by the high-ionization rates, $\sim\left(n_{e}\left\langle v_{e} \sigma\right\rangle\right)^{-1}$ corresponding to the collisional ionization of the lower states of ionization $Z$ for lighter elements, which increase as $\sim Z^{-3}$. Detailed atomic kinetics were included for all the ionization stages of each of the atomic components of polyacetal $(\mathrm{O}, \mathrm{C}$, and $\mathrm{H}$ ). The radiation transport was treated self-consistently, including the transport of line radiation, which in optically thin plasmas not completely ionized, usually dominates the continuum radiation loss rate by several orders of magnitude [39].

Wall ablation is very important in defining the plasma dynamics and is dominated by the absorption of plasma radiation, which can be computed accurately because the absorption properties of the wall material are well known in the spectral region below $100 \mathrm{~nm}$ in which the discharge emits the most [40]. However, there is incomplete thermophysical data available to describe organic polymers. Therefore, we followed the approach developed in laser ablation models that uses Arrhenius kinetics to model the decomposition of the wall material $[41,42]$. The model, that includes equations of state for the solid material and the plasma, makes use of the energies of vaporization and ionization to account for the transformation from solid to vapor to plasma.

The dynamics of the wall sustained plasma created in the evacuated microcapillary has an interesting and in some respects counterintuitive behavior. While the shadowgrams reveal a dynamics that originates at the walls and takes several tens of nanoseconds to reach the center of the microcapillary, the calculations show that the plasma reaches the axis of the capillary almost immediately, 2-5 ns after the beginning of the current pulse, when the rising current reaches values of the order of $100 \mathrm{~A}$. This is because both the characteristic hydroexpansion time and the Alfven time are of the order of several nanoseconds for the rare initial plasma with a temperature of $\sim 2 \mathrm{eV}$. Hence, the velocity of the absorption boundary observed in the shadowgrams of Fig. 2 should not be interpreted as the plasma mass speed. In fact, as discussed below, this velocity has no relationship with it. This illustrates that the interpretation of the shadowgrams corresponding to the microcapillary discharge can be far from obvious. Also, it was found that in spite of the relatively small peak current the plasma has a substantially high-electron temperature and degree of ionization.

The computed temporal evolution of the plasma parameters in the axial region of the microcapillary is illustrated in Fig. 5. The results were obtained assuming an approximately sinusoidal current pulse with a $1.5 \mathrm{kA}$ peak amplitude and 155 ns first half-cycle duration shown in Fig. 5(a), which very closely resembles that utilized in the experiments. Figure 5(b) shows the computed evolution of the electron temperature in the axis of the capillary. Initially the temperature rises very rapidly, exceeding $20 \mathrm{eV}$ during the first 10-20 ns of the current pulse. This initial temperature peak is a consequence of the very small electron density present at that moment $\left(n_{e} \approx 1 \times 10^{16} \mathrm{~cm}^{-3}\right)$, that results in the deposition of 

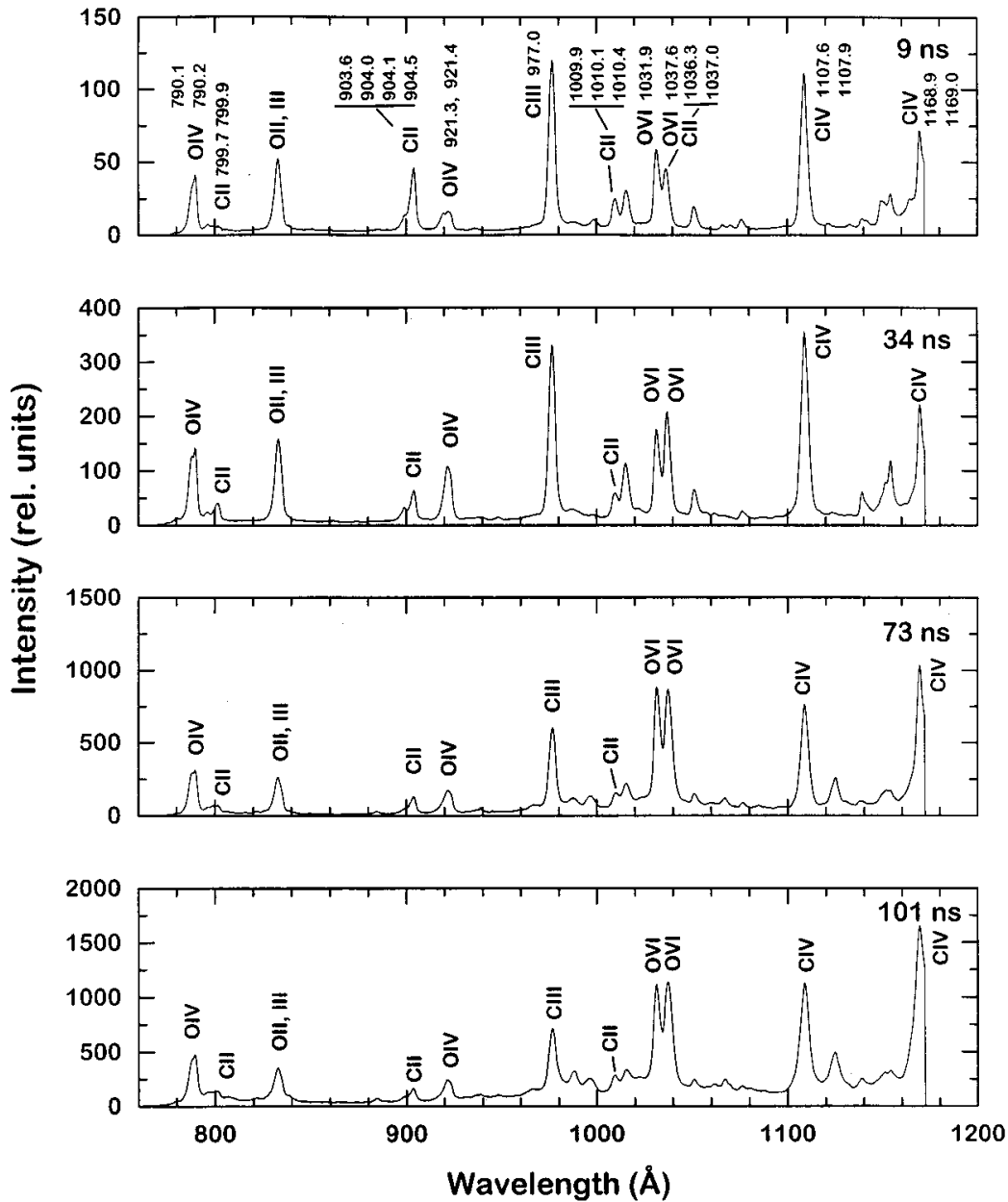

FIG. 4. Sequence of time-resolved spectra of the radiation self-emitted by the microcapillary discharge in the $78-117 \mathrm{~nm}$ spectral region. The time at which each spectrum was acquired with respect to the onset of the current pulse is indicated. a large fraction of the discharge input power into a relatively small number of particles. This is assisted by the fact that the electric conductivity in a completely ionized plasma is practically independent of the electron density, having only a weak dependence through the Coulomb logarithm [43]. The computed temperature is in agreement with the observation in the experiment of emission from $\mathrm{O}$ VI lines almost from the beginning of the discharge (taking into account $\sim 20 \mathrm{~ns}$ temporal resolution of the spectra). Steady-state ionization equilibrium calculations using a collisional-radiative model show that the electron temperature must be in the $T_{e}$ $\sim 15-20 \mathrm{eV}$ range for these ions to be present (Fig. 6). However, conditions inside the capillary are far from local thermodynamic equilibrium, which is achieved only in the heated boundary layer of the wall material. Instead, the ionization balance in this discharge is quasisteady state and follows the electron temperature with a characteristic time delay. The complete RADEX hydro and transient kinetic calculations reveal that, for a short period of time, $T_{e}$ is a factor $\sim 1.5$ larger than the steady-state value. The computations also show that after this short lasting initial maximum the temperature drops as a result of the permanent inflow of mass, which causes the density to steadily increase up to 3 $\times 10^{19} \mathrm{~cm}^{-3}$ [Fig. 5(d)]. Nevertheless, the mostly decaying trend of the electron temperature shows a local maximum near the peak of the current. A combination of multiple physical effects is responsible for such behavior of the electron temperature. First, the heating in the microcapillary is solely due to Joule dissipation. In this electric discharge the absorbed electromagnetic energy is distributed almost equally between the thermal energy of electron and ions, and potential energy associated with ionization. Heating due to the conversion of directed kinetic energy, which can be very important in ordinary $Z$ pinches, makes here only a small contribution to the electron temperature that does not exceed $1 \%$ during the entire discharge evolution.

Excluding the first $\sim 20 \mathrm{~ns}$, line radiation losses are substantially suppressed by the falling temperature and by the very high absorption of the ion lines. The total radiation losses integrated over all frequencies reach $\sim 15 \%$ of the discharge power at the time of maximum current, and $\sim 50 \%$ at the end of the first half-cycle of the current pulse. The strong absorption is due to the relatively large wavelengths of the transitions involved (300-800 $\AA$ ), and to the absence of any significant ion radial velocity gradient. The very large line absorption coefficient $\left(\sim 10^{5}-10^{6} \mathrm{~cm}^{-1}\right)$ combined with the large collisional quenching of the excited states of the predominant low- $Z$ ions $\left(\propto Z^{-3}\right)$ severely reduce the outflow of line radiation. In contrast, the central region of the plasma column is optically thin for both photorecombination and bremsstrahlung radiation. As a consequence, starting at $\sim 20$ ns after the beginning of the current pulse photorecombination represents the major loss mechanism, a situation which is typical of optically thick and recombining plasmas. Dielectronic recombination is substantially suppressed due to density effects [44]. In addition, in our case radiation does not constitute a complete energy loss, because the quanta with an energy of $\approx 10-45 \mathrm{eV}$ are very strongly absorbed in 


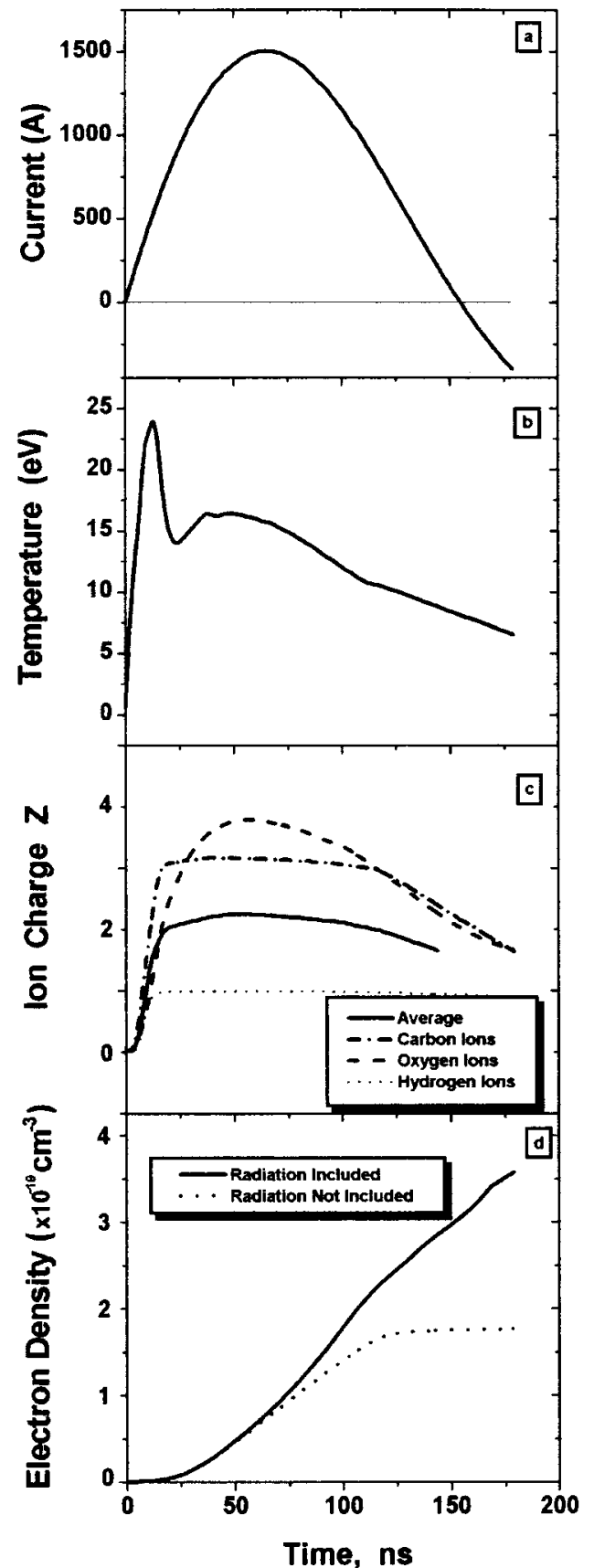

FIG. 5. Computed temporal evolution of the plasma parameters for the current pulse shown in frame (a). Frames (b)-(d) illustrate the variation of the on-axis values of the electron temperature, average ion charge of all atomic species present, and electron density, respectively.

a very thin wall layer nearest to the plasma, and following ablation a significant part of this energy returns to the plasma. The absorption coefficient of the atoms in the wall material reach the maximum value $\approx 1 \times 10^{-6} \mathrm{~cm}^{-1}$ in the wavelength region where the plasma emits the most. This large coefficient causes the majority of the radiation to be absorbed in a layer of just several monolayers thick, $\sim 10-$ $100 \AA$ of the wall material. This ablated layer is responsible for providing the material that once ionized constitutes the plasma that fills the microcapillary. To quantitatively emphasize the importance of plasma radiation-induced wall ablation in determining the electron density and the plasma dy-
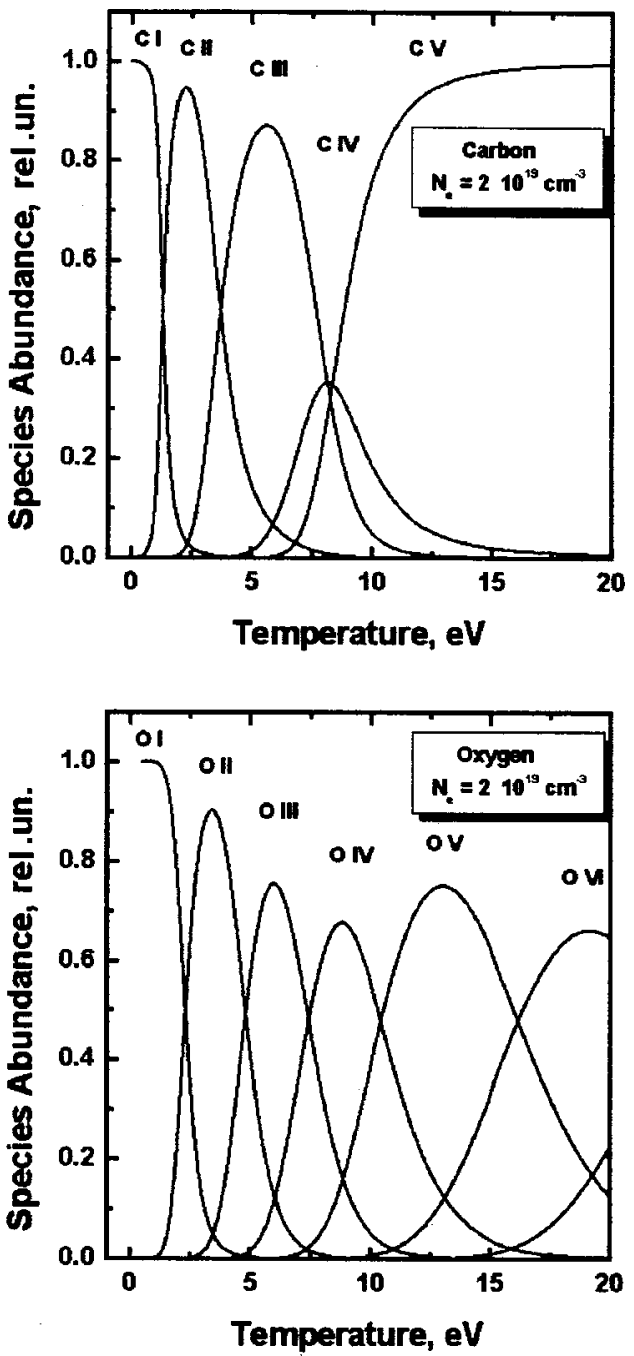

FIG. 6. Steady-state ionization balance for carbon (a) and oxygen ions (b) as a function of electron temperature at a fixed electron of $2 \times 10^{19} \mathrm{~cm}^{-3}$.

namics, Figure 5(d) shows the computed on-axis plasma density with and without radiation-induced evaporation included. We observe that after an initial rapid increase in density, due to material evaporated by electron heat conduction, plasma radiation $\left(\propto n_{e}^{2}\right)$ dominates the evaporation, increasing the density by a factor of $2-3$ by the end of the first half-cycle of the current pulse.

Another important effect that allows for the generation of the relatively high-electron temperature observed in this plasma is the saturation of the electron heat conductivity in the boundary layer with high-temperature gradient that separates the central and the cold wall plasma regions. Heat conductivity saturation effects have been intensively discussed for more than two decades in the literature, mainly with respect to laser-matter interactions [45-47]. As compared to laser produced plasmas, which include ions of higher charge $Z$ and large electric and spontaneous magnetic fields that can cause several different kinds of instabilities and inhomogeneities, the main reason of the saturation of the heat flux in the microcapillary plasmas is easier to separate. First, the plasma density is a few orders of magnitude smaller than in laser-created plasmas. Hence, the required condition for classical diffusive heat flow (Fourier law) with Knudsen number 


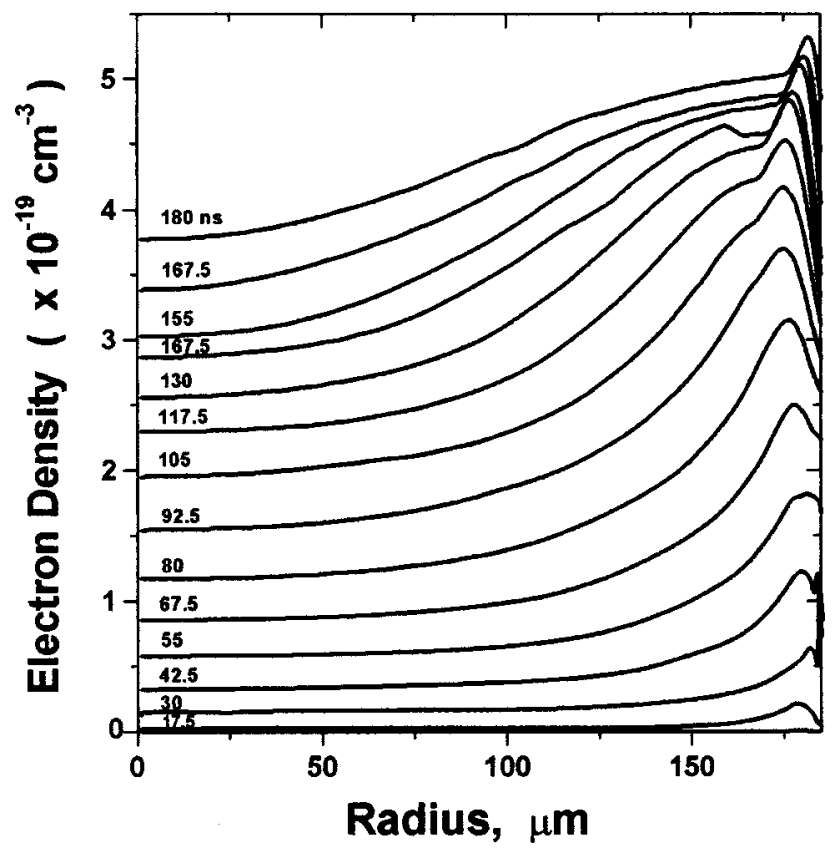

FIG. 7. Computed electron density profiles as a function of time measured from the beginning of the current pulse.

$\lambda_{e} / \lambda_{T}<10^{-2}[45,46]$, is easily violated because the ratio of electron mean-free-path, $\lambda_{e}$, to the temperature gradient length, $\lambda_{T}$, in the thin boundary layer near the wall is not sufficiently small. Up to the time of the current maximum this ratio is 10 to 30 times larger than the typical value required for classical diffusion. Other effects, such as the magnetic field associated with the current flow are insufficiently strong to significantly influence the heat conductivity (the resulting $\omega_{e} \tau_{e}<0.1$, where $\omega_{e}$ is the electron gyrotron frequency and $\tau_{e}$ is the electron collision time [43]). In our heat conductivity calculations we used the classical expression for a saturated flux treated in terms of the electron thermal speed $v_{e}$ and the so-called free-streaming factor $f \sim 0.15, q_{\text {sat }}$ $=f n_{e} T_{e} v_{e}$ [46], and obtained good agreement with the parameters observed in the experiment. A decrease of this factor to $f \sim 0.05$ still allowed agreement within the experimental error bars. An even larger heat flux, due to, for example, acoustic turbulence, is unlikely because in the periphery of the plasma $Z T_{e} / T_{i}<1-2$. Such a temperature ratio is not high enough to allow for the growth of such instability despite the relatively large electron drift velocity as compared to the sound speed $c_{s}\left[j / n_{e} \approx(5-10) c_{s}\right]$ [47]. In all other aspects the plasma kinetics remains classical, and the assumption of one-dimensional hydrodynamics is quite adequate.

One of the most important properties of microcapillaries plasmas is their ability to create a concave electron-density profile for applications such as laser-pulse waveguiding for weak field particle accelerators and $\mathrm{x}$-ray laser development. The computed density profiles, as a function of time, are shown in Fig. 7. The electron density increases significantly towards the walls, creating a plasma column with maximum index of refraction on axis that constitutes a plasma waveguide. The observed concave electron-density profile with a minimum on axis is formed due to several reasons. As it was mentioned above, in this small current discharge the magnetic $J \times B$ force is always smaller than the thermal pressure, and there is no significant kinetic motion associated with magnetic forces. Electron and ion pressure gradients have opposite signs and practically cancel each other at all locations. As a result, the plasma is sustained by ion counterpressure of the solid wall and is slowly contracted by the permanent inflow of evaporated material. Due to relatively large capillary length $L$ and short pulse duration, the end effects associated with the escape of the plasma into the vacuum do not play a substantial role, at least during the $\sim 150$ ns duration of first half-cycle of the current cycle. This is because $L \gg \tau c_{s}$, where $c_{s} \sim 5 \times 10^{5} \mathrm{~cm} / \mathrm{s}$ is the typical average speed of sound. As shown in Fig. 7, the plasma density on axis continuously increases as a function of time and is computed to reach values in excess of $\sim 3 \times 10^{19} \mathrm{~cm}^{-3}$ [48]. The lower density, and the smaller conductive and radiative cooling on axis, causes increased heating in that region. This in turn works as a positive feedback, resulting in an increased electrical conductivity, larger current density on axis, and increased Ohmic heating on axis. The result is the electron temperature distribution shown in Fig. 8, which decreases as a function of radius towards the wall. Due to pressure balance the density forms a profile with the opposite shape.

The lower electron temperature in the periphery of the plasma defines an annular region with a high concentration of C I, O I, and C II ions, all of which can be photoionized by the $26.5 \mathrm{eV}$ photons of the probe laser beam. A relatively small increase in the concentration of these ions can cause a sharp increase in the attenuation of the soft x-ray laser probe due to strong absorption caused by photoionization. This is the reason for the sharp boundary observed between the opaque and transparent regions in the shadowgrams of Fig. 2 and in the computed transmission profiles of Fig. 9. Free-free absorption is computed to make only a small contribution of less than $\sim 1 \%$ to the absorption of the probe beam. In the central region of the plasma the soft x-ray laser transverses the plasma without significant absorption, resulting in the observed flattop shadowgrams. As time evolves and the temperature on the axis drops, recombination causes an increase in the axial density of the weakly ionized atoms, which due to photoionization, strongly attenuate the laser beam. Computation of the spatial distributions of the concentration of the different ionic species indicates that the sharp absorption edge in the shadowgrams is mostly defined by the concentration of $\mathrm{C}$ II ions, that exist at higher temperatures than $\mathrm{O}$ I [see Figs. 6(a) and 6(b)] and that appear earlier in the axial region of the capillary when the plasma cools. At the abovementioned electron densities a fractional abundance of just $5 \%$ of these ions is sufficient to totally attenuate the probe beam. This absorption starts in the colder and denser periphery of the plasma column and propagates towards the axis, causing the observed size of both the region transparent to the soft $\mathrm{x}$-ray laser and self-emission region to decrease (Figs. 9 and 10). For comparison, the measured evolution of the boundary between the absorbent and transparent region of the plasma is illustrated by circles in Fig. 9. Finally, towards the end of the first half-cycle of the current, as the density on axis increases and the degree of ionization decreases due to recombination, the increased absorption causes the nearly complete attenuation of the probe beam observed in the experiment. The radial plasma flow, which after the first $\sim 5 \mathrm{~ns}$ is always subsonic, is almost completely 


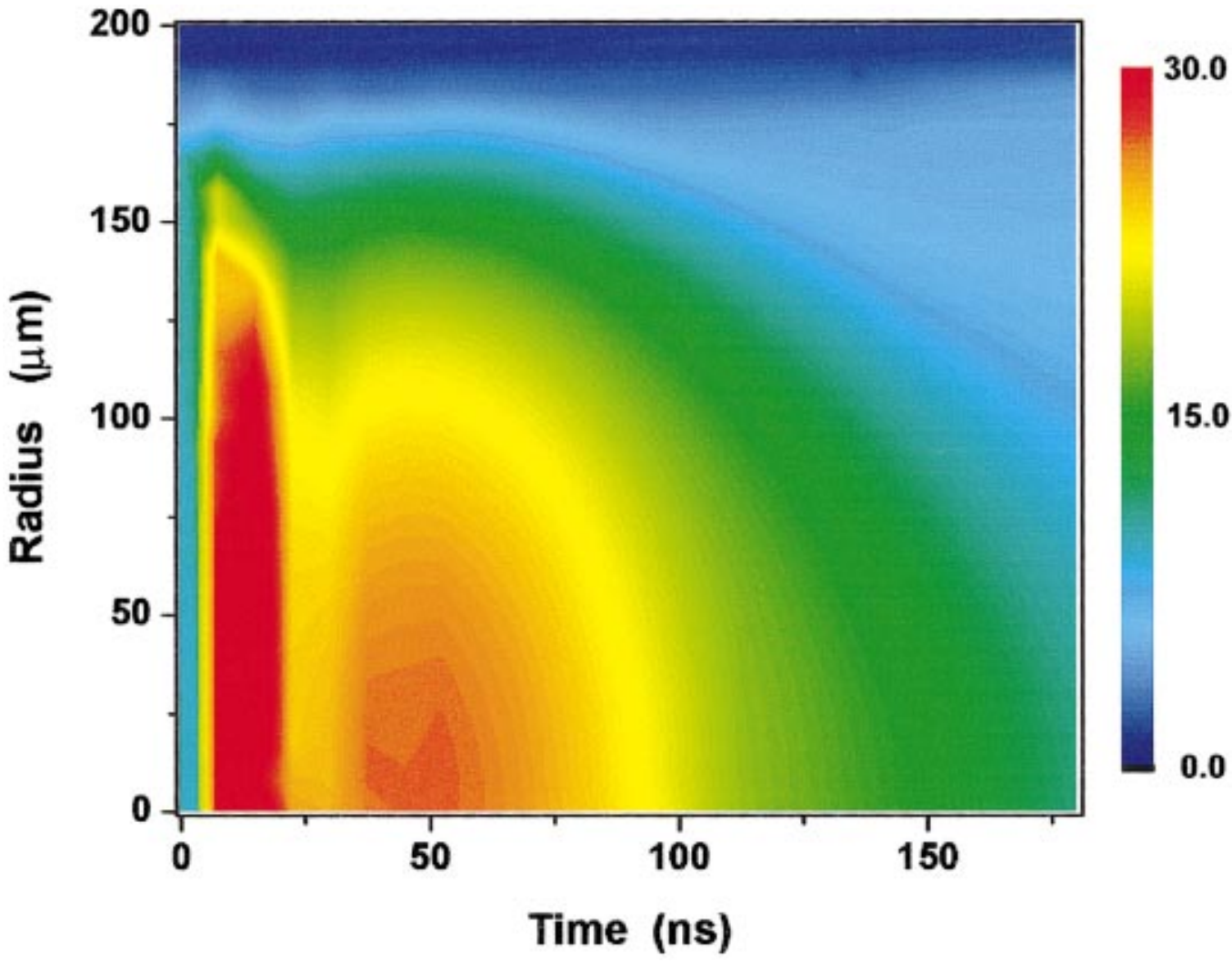

FIG. 8. (Color) Computed spatiotemporal distribution of the electron temperature. Other parameters are the same as in Fig. 5.

absent at this time. In contrast, the speed derived from the slope of the data points in Fig. 9 has a maximum of $\sim 10^{5} \mathrm{~cm} / \mathrm{s}$ at the end of first half-cycle of the current pulse. Hence, the model shows that the velocity of the boundary of the absorbing region has no direct relationship with the plasma velocity.
The results of the calculation of the size of the soft $\mathrm{x}$-ray emission source is shown in Fig. 10. Line radiation makes the main contribution to the emission in the initial phase of the discharge, when the plasma is transparent and rare, while photorecombination and dielectronic recombination are computed to be important in the latter part of the current pulse. In

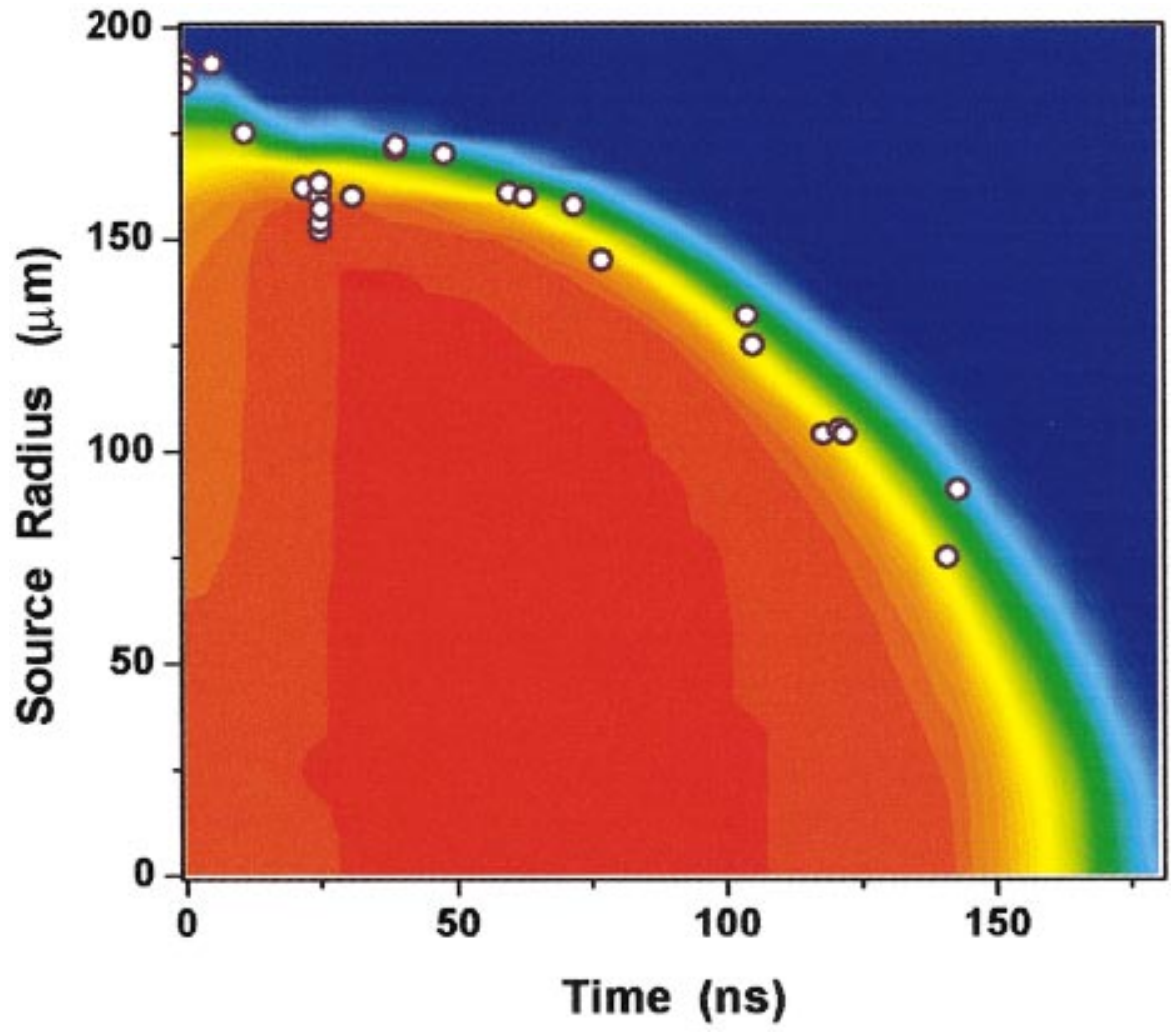

FIG. 9. (Color) Computed distribution of the soft $\mathrm{x}$-ray laser probe beam transmission at the exit of the microcapillary as a function of time. The values are normalized to the color scale of Fig. 8. The circles illustrate the measured boundary between the absorbing and transparent regions of the plasma. 


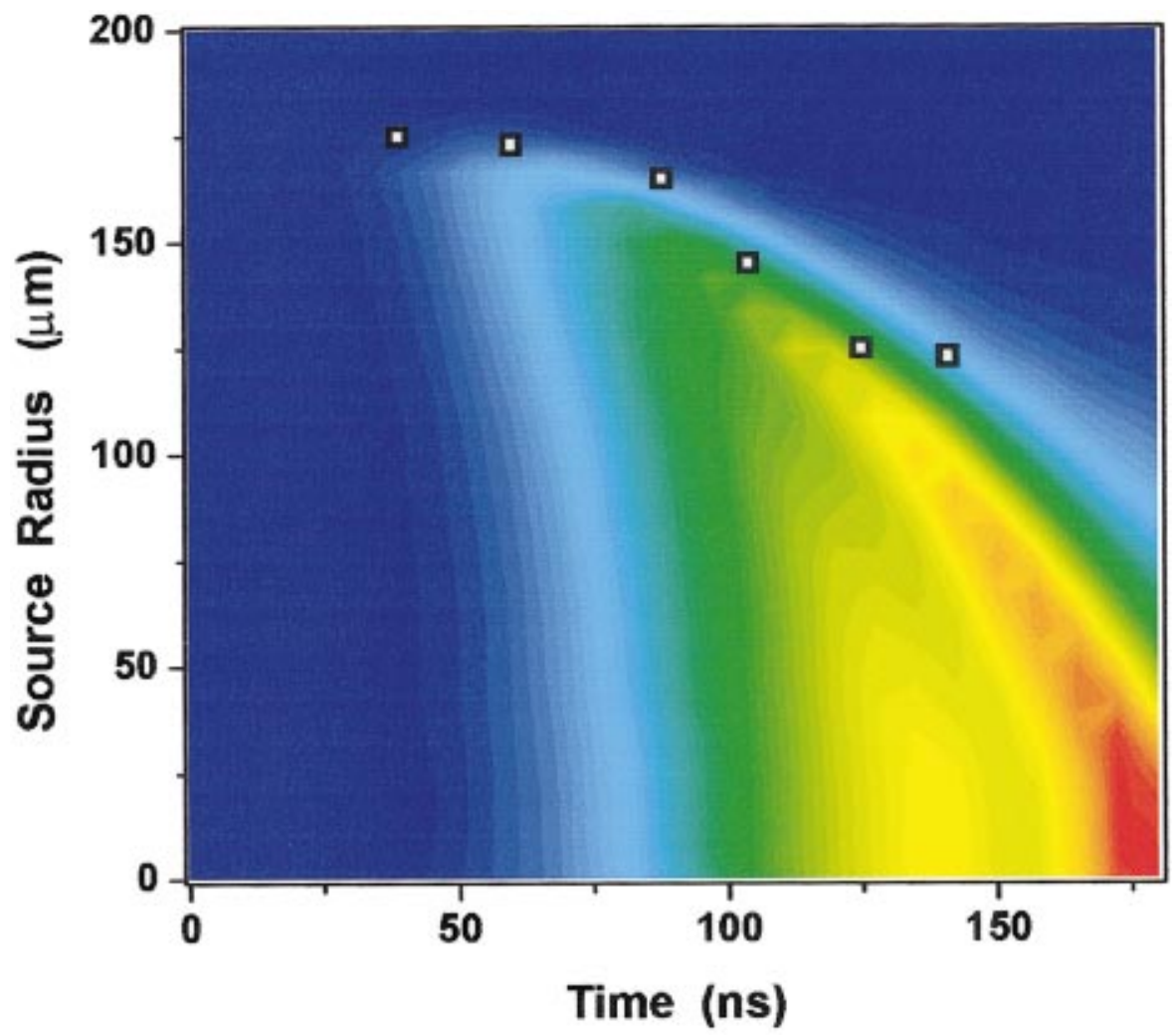

FIG. 10. (Color) Computed space and time evolution of the soft $\mathrm{x}$-ray/VUV radiation emitted by the plasma. For direct comparison with experiment the emission intensity was corrected by the spectral response of the detector. The maximum value is normalized to the color scale of Fig. 8. The squares indicate the measured radius of the emitting region.

comparison, the contribution of bremsstrahlung radiation is only $\sim 4 \%$. The radiated intensity integrated over the exit area of the capillary reaches its maximum approximately 110 ns after the beginning of the current, when the density on axis is already high, while the power density radiated at the capillary axis has a maximum later in time, at the end of the discharge (Fig. 10). Contrary to the absorption profiles, which have a flattop over the whole time history, the emission profiles change shape as the discharge evolves. The experimental result of Fig. 3 and the computations results illustrated in Fig. 10 show that early in the current pulse the emission profiles have distinctive maxima near the wall, where due to the concave profile the density is high. Towards the end of the first half-cycle of the current pulse, when the temperature of the peripheral region substantially drops to $3-5 \mathrm{eV}$, the computed emission profile also acquires a bell shape of larger size than the transparent region observed in the shadowgrams, in agreement with the experiment.

\section{CONCLUSIONS}

In summary, we have studied the dynamics of a plasma column created by discharge ablation of the walls in an evacuated microcapillary. Soft x-ray laser plasma shadowgrams and VUV self-emission measurement techniques combined with numerical modeling provided a self-consistent view of the dynamics of this plasma. The results show that the microcapillary plasma is initially nonuniform, but that it rapidly evolves into a highly symmetric column with minimum electron density and maximum temperature on-axis. The high symmetry of these plasma columns with concave electron profiles confirms the good properties of these discharges as plasma waveguides for laser radiation. Model computations successfully reproduced and explained the observation data. It was found that the evolution of sharp soft $\mathrm{x}$-ray absorption boundary observed in the experiments, which starts at the walls and propagates towards the axis, is dominated by photoionization of weakly ionized species. The evaluation of the different processes that characterize the plasma dynamics enabled us to distinguish this type of microcapillary from $Z$ pinches, classifying it into a wallsustained plasma. The plasma heating is solely due to Joule dissipation and the heating due to conversion of kinetic energy, which is important in $Z$ pinches, here makes only a very small contribution. The reduction of radiation losses caused by the large spectral line opacities, the saturation of the electron heat flux to the walls, and the absence of significant hydrodynamic losses from the capillary ends results in relatively large temperatures on axis (15-20 eV) and highionization states up to $\mathrm{O}$ VI at relatively small excitation currents. The results also show that plasma shadowgraphy with tabletop soft x-ray lasers can, in combination with model computations, help to elucidate the dynamics of dense plasmas.

\section{ACKNOWLEDGMENTS}

This work was supported by the U.S. Department of Energy under Grant No. DE-FG03-98DP00208 and the National Science Foundation under Grant No. ECS-9713297. Part of this work was performed under the auspices of the U.S. Department of Energy by the University of California Lawrence Livermore National Laboratory under Contract No. W-7405-Eng-48. M.C.M. acknowledges the support of CONICET. We also gratefully acknowledge support from the W. M. Keck Foundation. 
[1] H. Conrads, Z. Phys. 444, 200 (1967).

[2] P. Bogen, H. Conrads, G. Gatti, and W. Kohlhaas, J. Opt. Soc. Am. 58, 203 (1968).

[3] R. A. McCorkle, Appl. Phys. A: Solids Surf. 26, 261 (1981).

[4] M. C. Marconi and J. J. Rocca, Appl. Phys. Lett. 54, 2180 (1989).

[5] J. J. Rocca, M. C. Marconi, and F. G. Tomasel, IEEE J. Quantum Electron. 29, 182 (1993).

[6] J. J. Rocca, O. D. Cortazar, B. Szapiro, K. Floyd, and F. G. Tomasel, Phys. Rev. E 47, 1299 (1993).

[7] F. G. Tomasel, J. J. Rocca, O. D. Cortazar, B. T. Szapiro, and R. W. Lee, Phys. Rev. E 47, 3590 (1993).

[8] C. A. Morgan, H. R. Griemond, and R. C. Elton, Phys. Rev. E 49, 2282 (1994).

[9] F. T. Tomasel, J. J. Rocca, and V. N. Shlyaptsev, IEEE Trans. Plasma Sci. 24, 49 (1996).

[10] J. J. Rocca, V. N. Shlyaptsev, F. G. Tomasel, O. D. Cortazar, D. Hartshorn, and J. L. A. Chilla, Phys. Rev. Lett. 73, 2192 (1994).

[11] J. J. Rocca, D. P. Clark, J. L. A. Chilla, and V. N. Shlyaptsev, Phys. Rev. Lett. 77, 1476 (1996).

[12] F. G. Tomasel, J. J. Rocca, V. N. Shlyaptsev, and C. D. Macchietto, Phys. Rev. E 55, 1437 (1997).

[13] B. R. Benware, C. H. Moreno, D. J. Burd, and J. J. Rocca, Opt. Lett. 22, 796 (1997).

[14] B. R. Benware, C. D. Macchietto, C. H. Moreno, and J. J. Rocca, Phys. Rev. Lett. 81, 5804 (1998).

[15] Y. Ehrlich, C. Cohen, A. Zigler, J. Krall, P. Sprange, and E. Erasay, Phys. Rev. Lett. 77, 4186 (1996).

[16] J. J. Rocca, F. G. Tomasel, M. C. Marconi, J. L. A. Chilla, C. H. Moreno, B. R. Benware, V. N. Shlyaptsev, J. J. Gonzalez, and C. D. Macchietto, Proc. SPIE 3156, 164 (1997).

[17] D. Kaganovich, A. Ting, C. I. Moore, A. Zigler, H. R. Burris, Y. Ehrlich, R. Hubbard, and P. S. Sprangle, Phys. Rev. E 59, R4769 (1999).

[18] K. A. Janulewicz, F. Bortolotto, P. J. Warwick, M. P. Kalashnikov, V. N. Shlyaptsev, W. Sandner, J. J. Rocca, and P. V. Nickles, Proc. SPIE 3776, 37 (1999).

[19] A. Golstov, A. Morozov, S. Suckewer, R. Elton, U. Feldman, K. Krushelnick, T. Jones, C. Moore, J. Seely, S. Sprangle, A. Ting, and A. Zigler, Proc. SPIE 3776, 126 (1999).

[20] T. Hosakai, M. Kando, H. Dewa, H. Kotaki, S. Kando, N. Hasegawa, N. Nakajima, and H. Horioka, Opt. Lett. 25, 10 (2000).

[21] D. J. Spence, P. D. S. Burnett, and S. M. Hooker, Opt. Lett. 24, 993 (1999).

[22] D. Korobkin, C. H. Nam, S. Suckewer, and A. Golstov, Phys. Rev. Lett. 77, 5206 (1996).

[23] D. Korobkin, A. Goltsov, A. Morozov, and S. Suckewer, Phys. Rev. Lett. 81, 1607 (1998).

[24] C. G. Durfee III and H. M. Milchberg, Phys. Rev. Lett. 71, 2409 (1993).

[25] C. G. Durfee III, J. Lynch, and H. M. Milchberg, Opt. Lett. 19, 1937 (1994).

[26] C. G. Durfee III, J. Lynch, and H. M. Milchberg, Phys. Rev. E 51, 2368 (1995).

[27] H. M. Milchberg, C. G. Durfee III, and J. Lynch, J. Opt. Soc. Am. B 12, 731 (1995).

[28] T. Tajima and J. M. Dawson, Phys. Rev. Lett. 43, 267 (1979); P. Sprangle et al., Appl. Phys. Lett. 53, 2146 (1988).
[29] C. D. Macchietto, B. R. Benware, and J. J. Rocca, Opt. Lett. 24, 1115 (1999).

[30] F. Lowenthal, R. Tommasini, and J. E. Balmer, Phys. Rev. A 59, 1577 (1999).

[31] J. Dunn, Y. Li, A. L. Osterheld, J. Nilsen, S. J. Moon, K. B. Fournier, J. R. Hunter, A. Y. Faenov, T. A. Pikuz, and V. N. Shlyaptsev, Proc. SPIE 3776, 2 (1999).

[32] R. Cauble, L. B. Da Silva, J. T. W. Barbee, P. Celliers, J. C. Moreno, and A. S. Wan, Phys. Rev. Lett. 74, 3816 (1995).

[33] P. Celliers, T. W. Barbee, Jr., R. Cauble, L. B. Da Silva, C. D. Decker, D. H. Kalandar, M. H. Key, R. A. London, J. C. Moreno, R. Snavely, J. E. Trebes, S. Wan, and F. Weber, Proc. SPIE 3156, 135 (1997).

[34] C. H. Moreno, M. C. Marconi, V. N. Shlyaptsev, and J. J. Rocca, IEEE Trans. Plasma Sci. 27, 6 (1999).

[35] A. V. Vinogradov and V. N. Shlyaptsev, Kvant. Electron. (Moscow) 10, 509 (1983) [Sov. J. Quantum Electron. 13, 298 (1983)]; 10, 2325 (1983) [13, 1511 (1982)]; Yu. V. Afanasiev et al., Sov. J. Laser Res. 10, 1 (1989); V. N. Shlyaptsev, A. V. Gerusov, A. V. Vinogradov, J. J. Rocca, O. D. Cortazar, F. Tomasel, and B. Szapiro, Proc. SPIE 2012, 99 (1993).

[36] V. N. Shlyaptsev, J. J. Rocca, and A. L. Osterheld, Proc. SPIE 2520, 365 (1995).

[37] P. V. Nickles, V. N. Shlyaptsev, M. Kalashnikov, M. Schnurer, I. Will, and W. Sandner, Phys. Rev. Lett. 78, 2748 (1997); J. Dunn, A. L. Osterheld, R. Shepherd, W. E. White, V. N. Shlyaptsev, and R. E. Stewart, ibid. 80, 2825 (1998).

[38] J. Von Neumann and R. D. Richtmeyer, J. Appl. Phys. 21, 232 (1949).

[39] D. E. Post, R. V. Jensen, and C. B. Tarter, At. Data Nucl. Data Tables 20, 397 (1977); A. V. Vinogradov and V. N. Shlyaptsev, Kvant. Electron. (Moscow) 14, 5 (1987) [Sov. J. Quantum Electron. 17, 1 (1987)].

[40] B. L. Henke, E. M. Gullikson, and J. C. Davis, At. Data Nucl. Data Tables 54, 181 (1993).

[41] Yu V. Afanasiev and O. N. Krokhin, High-Temperature and Plasma Phenomena at the Influence of High-Power Radiation with Matter (Mir, Moscow, 1974) (in Russian).

[42] Science, edited by A. A. Samokhin, Proceedings of General Physics Institute (Nauka, Moscow, 1988) (in Russian), Vol. 13.

[43] L. Spitzer, Physics of Fully Ionized Gases (Wiley, New York, 1957); S. I. Braginskii, Transport Processes in a Plasma, edited by M. A. Leontovich, Review of Plasma Physics (Consultants Bureau, New York, 1963).

[44] V. P. Shevelko and L. A. Vainshtein, Atomic Physics for Hot Plasmas (IOP, Bristol, 1993).

[45] C. E. Max, C. F. McKee, and W. C. Mead, Phys. Fluids 23, 1620 (1980); N. G. Basov, A. S. Shikanov, G. V. Sklizkov, Yu. A. Zakharenkov, D. W. Sweeney, and D. T. Attwood, Fiz. Plazmy 6, 1167 (1980) [Sov. J. Plasma Phys. 6, 642 (1980)].

[46] S. A. Khan and T. D. Rognlien, Phys. Fluids 24, 1442 (1981); R. H. Cohen and T. D. Rognlien, Contrib. Plasma Phys. 34, 198 (1994).

[47] V. Bychenkov and V. P. Silin, Zh. Eksp. Teor. Fiz 82, 1886 1982 [Sov. Phys. JETP 55, 1086 (1982)].

[48] A. L. Osterheld et al., Proceeding of the 6th International Conference on X-ray Lasers, Kyoto, edited by Y. Kato, H. Takuma, and H. Daido (IOP, Bristol, 1998). 